\title{
A Performance Analysis of Two Approximate Adaptive Designs
}

\author{
Mark French, Member, IEEE
}

\begin{abstract}
The performance of function approximator based adaptive control designs may scale badly with approximator dimension [1]. For a simple system class, both projection based designs and multi-resolution approximation based designs have been shown to have good scaling properties w.r.t. to LQ costs. Here we show that by considering a cost functional with penalties on the control rate, the multi-resolution approximatior based design can outperform the projection based design. Generalisations are briefly discussed.
\end{abstract}

\section{INTRODUCTION}

Function approximators have been widely used in adaptive control design since the publication of [2], [3], see for example the recent monographs [4], [5], [6] for an overview and [7] as a representative recent paper. The role of function approximators is to replace function uncertainties with parametric uncertainties in the system equations so that standard parametric adaptive techniques can be utilized. Once the approximator structure and dimension has been determined, the designs are essentially those from robust adaptive theory, where techniques such as dead-zones, projection, $\sigma$ modification are used to handle the minimal approximation error (which is thought of as a disturbance). The only remaining stability issue is to ensure that the system never leaves the (typically compact) region where the minimal function approximation error can be small, or alternatively, that the system can be guaranteed to be stable even if this region is left. Typically this is ensured by either limiting the uncertainty, increasing the system gains, or by using robust terms or sliding mode techniques for stability in the large.

In this paper we focus on the choice of function approximator, and its consequences for closed loop performance. Given an approximator structure, the required size $(m)$ of the approximator is determined as a function of the system smoothness $(\beta)$ and the required minimal approximation error $(\epsilon)$. Typically $\beta$ represents a bound in a Sobolev space or a Lipschitz constraint, $\mathcal{W}^{m}$ is the parameter space whose dimension is an increasing function of $m$, and the approximation error $\epsilon$ is measured uniformly over some compact set (eg. in $C(\Omega)$, $\Omega$ compact). The relationship between $m, \beta$ and $\epsilon$ is described by a dimension function:

$$
\rho: \operatorname{dom}(\rho) \rightarrow \mathbb{N}, \quad \rho(\beta, \epsilon)=m
$$

An example of a dimension function is given by Jackson's theorem for polynomial approximation in $C[a, b]$, where $m$ is the polynomial degree, $\beta$ is a Lipschitz constraint and the dimension function is given by $\rho=\frac{\beta(b-a)}{3 \epsilon}$.

M. French is with the School of Electronics and Computer Science, University of Southampton.
Typically, the smoothness $\beta$ of the system is only known conservatively, ie. we know $\hat{\beta}>>$, consequently, we will generally use a function approximator of overly large dimension in the controller. The fundamental question is as to whether this has any detrimental effect.

More precisely, suppose we have a uncertain system $\Sigma(\beta)$, whose smoothness is only known up to an upper bound $\hat{\beta}$ of $\beta$. A controller $\Xi(\hat{\beta})$ is designed depending on $\hat{\beta}$. Let $\mathcal{P}([\Sigma(\beta), \Xi(\hat{\beta})])$ denote a closed loop cost (eg. a worse case LQ cost, see $\mathcal{P}_{1}$ in (13), (15) below). A prerequisite for a sensible control design is the idea of $\mathcal{P}$ stability, ie. that:

$$
\mathcal{P}([\Sigma(\beta), \Xi(\hat{\beta})])<\infty \quad \forall \hat{\beta} \geq \beta .
$$

A further desirable property for the controller is to establish resolution scaleability, ie the property that for all fixed $\beta$ :

$$
\limsup _{\hat{\beta} \rightarrow \infty} \mathcal{P}([\Sigma(\beta), \Xi(\hat{\beta})])<\infty .
$$

This is the property that for large enough $\hat{\beta}$ (typically for any $\hat{\beta} \geq \beta$ ), there is a uniform performance bound.

On the other hand if the controller is not resolution scaleable, ie. there is no such uniform bound, then clearly the choice of approximator dimension is critical, and in particular, as the knowledge of the smoothness $\hat{\beta}$ becomes conservative, then the performance deterioates. In fact, we will give an example of a class of $\mathcal{P}$ stable controllers with the resolution divergence property:

$$
\liminf _{\hat{\beta} \rightarrow \infty} \mathcal{P}([\Sigma(\beta), \Xi(\hat{\beta})])=\infty \quad \forall \beta \geq 0 .
$$

In [1] an example was constructed which showed that there is a large class of standard function approximator designs which are resolution divergent w.r.t. LQ costs. Hence it is significant to find designs which are resolution scaleable. There have been two qualitatively different proposals to ensure resolution scaleability for function approximator designs w.r.t. LQ costs. The first [8] uses projection modifications and high adaption gains, the second is based on a multi-resolution function approximator [1]. The goal of this paper is to compare these two proposals, and in particular to show that the latter design outperforms the former when the cost also includes penalties on the control rate $\dot{u}$.

This paper also establishes resolution divergence for some standard approximators in a much simpler setting than in the original paper [1]. Much of the technical complexity of [1] is avoided by changing the LQ cost to a cost incorporating a penalty on $\dot{u}$.

The results are developed on a scalar system. This is a deliberate decision of the authors for the following reasons. 
One contribution of this paper is to demonstrate the mechanism by which unmodified and projection modified designs are resolution divergent when the control rate is penalised. It is clear that a similar mechanism occurs in more general designs for wider classes of systems, and to illustrate the mechanism in a more general framework would add little other than notational complications. The second main contribution of this paper is to demonstrate that the multi-resolution designs are resolution scaleable. Again the mechanism by which this occurs is clear from the proof and it is apparent how the technique should generalise (see for example [5]). To present the resolution scaling results in a more general setting would obscure the main idea, namely that multi-resolution designs have inherent resolution scaling properties w.r.t. a wide range of cost functionals. We briefly discuss the extensions of the results in the final section of the paper. The main notation is detailed at the end of the paper.

\section{Problem Formulation}

\section{A. Approximation Theory}

To define an approximate adaptive design and to precisely formulate the problem investigated in this paper, we first define some approximation theoretic notions. A $\mathcal{F}$ smoothness class is a nested set of subsets $\left\{K_{\beta}\right\}_{\beta>0}$ of a function space $\mathcal{F}$, ie. $K_{\beta}^{\prime} \subset K_{\beta}$ when $\beta^{\prime} \leq \beta$, and where $\mathcal{F}=\cup_{\beta>0} K_{\beta}$. Typically a smoothness class is specified by Lipschitz constraints, or by bounds in Sobolev spaces as is typical in approximation theory. A model class is a sequence of model bases $\left\{\phi^{m}\right\}_{m>1}$, $\phi^{m}: \mathbb{R} \rightarrow \mathcal{W}^{m}$ and where $\mathcal{W}^{m}$ is a Euclidean space called the weight space of the $m$ th model. Typically $\operatorname{dim} \mathcal{W}^{m}$ will be a divergent function of $m$. Generic approximation theory furnishes us with a dimension function $\rho_{\mathcal{F}}: \operatorname{dom}\left(\rho_{\mathcal{F}}\right) \rightarrow \mathbb{N}$ where $\operatorname{dom}\left(\rho_{\mathcal{F}}\right) \subset \mathbb{R}_{+} \times \mathbb{R}_{+}$. For a smoothness parameter $\beta$, approximation bound $\epsilon$ and normed function space $\mathcal{F}$, the dimension function satisfies:

$$
\forall f \in K_{\beta} \forall m \geq \rho(\beta, \epsilon) \exists \theta \in \mathcal{W}^{m} \text { s.t. }\left\|f-\theta^{T} \phi^{m}\right\|_{\mathcal{F}}<\epsilon .
$$

If $\mathcal{D}=\mathbb{R}_{+} \times \mathbb{R}_{+}$then the model class is said to be an $\left(\mathcal{F},\left\{K_{\beta}\right\}_{\beta \geq 0}\right)$ approximate model class.

Definition 2.1: A multi-resolution model class is a model class $\left\{\phi^{m}\right\}_{m \geq 1}$ with the structure

$$
\phi^{m}=\left[\tilde{\phi}^{1}\left|\tilde{\phi}^{2}\right| \ldots \mid \tilde{\phi}^{m}\right]
$$

where $\left\{\tilde{\phi}^{k}: \mathbb{R} \rightarrow \mathcal{W}^{k}\right\}_{k \geq 1}$ is a model class, called the base class.

Note that this definition imposes considerable structure on the model class, it requires that the higher resolution models are simply basis extensions of the lower resolution models, hence standard approximation bases such as Taylor series, Fourier series, wavelets etc. are all of the appropriate form. Basis functions from any $\left(\mathcal{F},\left\{K_{\beta}\right\}_{\beta \geq 0}\right)$ approximation class can be utilized as the base class, for if $\left\{\tilde{\phi}^{k}\right\}_{k \geq 1}$ is a $\left(\mathcal{F},\left\{K_{\beta}\right\}_{\beta \geq 0}\right)$ approximation class then so is $\left\{\phi^{m}\right\}_{m \geq 1}$. However, note that $\phi^{m}$ is of much higher dimensional than $\tilde{\phi}^{m}$ as it is the concatenation of all the lower resolution models.

\section{B. System}

Let $\mathcal{U}, \mathcal{Y}$ be function spaces representing the input and output signal spaces. A system is denoted by $\Sigma$, and lies in $\mathcal{S}$, the set of all causal operators $\mathcal{U} \rightarrow \mathcal{Y}$. A controller is denoted by $\Xi$ and lies in $\mathcal{C}$, the set of all causal operators $\mathcal{Y} \rightarrow \mathcal{U}$. We define an interconnection $[\Sigma, \Xi]$ of a system $\Sigma$ and controller $\Xi$ as $[\Sigma, \Xi]=(y, u)$ where $y, u$ are the closed loop signals (ie. the solutions of $\Sigma u=y, u=\Xi y$ ).

For notational simplicity and conceptual clarity we consider scalar systems $\Sigma_{y_{0}}(f)$ of the form:

$$
\Sigma_{y_{0}}(f): \dot{y}=f(y)+u, \quad y(0)=y_{0} \in \mathbb{R}
$$

An initial condition set is defined as:

$$
\mathcal{Y}_{0}=\left\{y_{0} \in \mathbb{R}|| y_{0} \mid \leq \gamma_{0}\right\},
$$

and define $\Sigma_{\mathcal{Y}_{0}}=\left\{\Sigma_{y_{0}}(\{f\}) \mid y_{0} \in \mathcal{Y}_{0}\right\}$.

We define an uncertainty set $\Delta(\beta)$ to be of the form:

$$
\begin{aligned}
\Delta(\beta) & =K_{\beta} \cap \Delta\left(L^{\infty}\left(\Omega ; w_{\infty}\right), \delta_{\infty}\right) \\
& =\left\{f \in K_{\beta}|| f(x) \omega_{\infty}(x) \mid \leq \delta_{\infty} \forall x \in \Omega\right\},
\end{aligned}
$$

where $\left\{K_{\beta}\right\}_{\beta \geq 0}$ is a $C(\Omega, \mathbb{R})$ smoothness class, $\Omega=[-W, W]$ is compact and where $0 \leq \delta_{\infty}<\infty$.

\section{Performance}

The control task is to asymptotically track a given reference trajectory to within a specified accuracy. In addition all closed loop signals should be kept bounded. Performance will then be judged w.r.t. to a cost functional which penalises transient signals.

So, given a reference trajectory $y_{\text {ref }} \in C^{1}\left(\mathbb{R}_{+}, \mathbb{R}\right)$, we define a reference trajectory set as:

$$
\begin{aligned}
\mathcal{Y}_{\text {ref }}=\left\{y_{\text {ref }} \in C^{1}\left(\mathbb{R}_{+}, \mathbb{R}\right) \quad \mid\right. & \left\|y_{\text {ref }}\right\|_{L^{\infty}\left(\mathbb{R}_{+}\right)} \leq \gamma_{1}, \\
& \left.\left\|y_{\text {ref }}{ }^{(1)}\right\|_{L^{\infty}\left(\mathbb{R}_{+}\right)} \leq \gamma_{2}\right\}(10)
\end{aligned}
$$

where $\gamma_{1}, \gamma_{2} \geq 0$ are fixed numbers, known to the control design. The control task is to give a controller $\Xi\left(y_{\mathrm{ref}}\right)$ to achieve asymptotic $\Omega_{0}=[-\eta, \eta]$ tracking whilst keeping all signals bounded, in the sense of the following definition, (note the inner supremum is taken over all (non-unique) Fillipov solutions [9]):

Definition 2.2: A closed loop $\left(\Sigma_{\mathcal{Y}_{0}}(\Delta(\beta)), \Xi\left(\mathcal{Y}_{\text {ref }}\right)\right)$ is said to achieve asymptotic $\Omega_{0}$ tracking if:

$$
\begin{array}{lll}
\sup _{f \in \Delta(\beta)} \sup _{y_{0} \in \mathcal{Y}_{0}} & \sup _{y_{\text {ref }} \in \mathcal{Y}_{\text {ref }} \operatorname{solns}\left(\Sigma_{y_{0}}(f), \Xi\left(y_{\text {ref }}\right)\right)} \\
& \lim _{t \rightarrow \infty} \operatorname{dist}\left\{\left(y-y_{\text {ref }}\right)(t), \Omega_{0}\right\}=0 .
\end{array}
$$

The closed loop is said to be bounded if all closed loop signals lie in $L^{\infty}$.

The closed loop signals are taken to be the system and controller's state, input and output signals. So that the control task is achievable we impose the following constraint throughout the paper:

$$
\gamma_{0}+\gamma_{1}<W
$$

Performance of a closed loop is measured by a functional of the output and input signals:

$$
\mathcal{J}: \mathcal{Y} \times \mathcal{U} \rightarrow \mathbb{R}_{+}
$$


Throughout we consider four specific cost functionals which penalise the non-singular transient performance of the system and are given by:

$$
\begin{aligned}
& \mathcal{J}_{1}[y(\cdot), u(\cdot)]=\int_{\mathcal{T}_{\Omega_{0}}} x^{2}(t)+u^{2}(t) d t \\
& \mathcal{J}_{2}[y(\cdot), u(\cdot)]=\int_{\mathcal{T}_{\Omega_{0}}} x^{2}(t) d t+\sup _{t \geq 0}|u(t)| \\
& \mathcal{J}_{3}[y(\cdot), u(\cdot)]=\int_{\mathcal{T}_{\Omega_{0}}} x^{2}(t)+u^{2}(t) d t+\sup _{t \geq 0}|\dot{u}(t)| \\
& \mathcal{J}_{4}[y(\cdot), u(\cdot)]=\int_{\mathcal{T}_{\Omega_{0}}} x^{2}(t) d t+\sup _{t \geq 0}|u(t)|+\sup _{t \geq 0}|\dot{u}(t)|
\end{aligned}
$$

where

$$
x(\cdot)=y(\cdot)-y_{\mathrm{ref}}(\cdot), \quad \text { and } \quad \mathcal{T}_{\Omega_{0}}=\left\{t \geq 0 \mid x(t) \notin \Omega_{0}\right\} .
$$

Such a cost penalises the response of the system whilst $x(t) \notin \Omega_{0}=[-\eta, \eta]$, hence for a closed loop whose goal is to drive $x$ to $\Omega_{0}$, whilst keeping $y, u$ bounded, these costs are reasonable penalties on the transient signals.

Performance of a controller $\Xi$ will be measured in this paper with respect to a worst case cost, ie. $\mathcal{P}: P(\mathcal{S}) \times \mathcal{C} \rightarrow \mathbb{R}_{+},{ }^{1}$ where $P(\mathcal{S})$ denotes the power set of $\mathcal{S}$, and where:

$$
\begin{aligned}
& \mathcal{P}_{j}\left(\Sigma_{\mathcal{Y}_{0}}(\Delta), \Xi\right) \\
= & \sup _{f \in \Delta(\beta)} \sup _{y_{0} \in \mathcal{Y}_{0}} \sup _{y_{\mathrm{ref}} \in \mathcal{Y}_{\text {ref }} \operatorname{solns}\left(\Sigma_{y_{0}}(f), \Xi\left(y_{\mathrm{ref}}\right)\right)} \operatorname{Jup}_{j}[y(\cdot), u(\cdot)] \\
& 1 \leq j \leq 4 .^{2}
\end{aligned}
$$

\section{Controllers}

We now recall an important definition [10].

Definition 2.3: A $\mathcal{P}$ stable control design is a mapping $\Gamma: \mathbb{R}_{+} \rightarrow \mathcal{C}$ such that:

$$
\mathcal{P}\left(\left[\Sigma_{\mathcal{Y}_{0}}(\Delta(\beta)), \Gamma(\beta)\left(\mathcal{Y}_{\text {ref }}\right)\right]\right)<\infty, \quad \forall \beta \geq 0
$$

We are thus concerned with the behaviour of a class of controllers $\{\Gamma(\beta)\}_{\beta \geq 0}$ as specified by the design function $\Gamma$, which defines a (different) controller for each smoothness level $\beta$. Note that for any $\mathcal{P}$ stable design it follows that

$$
\mathcal{P}\left(\left[\Sigma_{\mathcal{Y}_{0}}\left(\Delta\left(\beta^{\prime}\right)\right), \Gamma(\beta)\left(\mathcal{Y}_{\text {ref }}\right)\right]\right)<\infty, \quad \forall \beta^{\prime} \leq \beta
$$

by the nested property of $\left\{K_{\beta}\right\}_{\beta>0}$.

In this paper, we are primarily concerned with the following two properties of $\mathcal{P}$ stable control designs:

Definition 2.4: A $\mathcal{P}$ stable control design $\Gamma: \mathbb{R}_{+} \rightarrow \mathcal{C}$ is said to be resolution scaleable if:

$$
\limsup \mathcal{P}\left(\left[\Sigma_{\mathcal{Y}_{0}}(\Delta(\beta)), \Gamma(\hat{\beta})\left(\mathcal{Y}_{\text {ref }}\right)\right]\right)<\infty, \quad \forall \beta \geq 0 .
$$

Definition 2.5: A $\mathcal{P}$ stable control design $\Gamma: \mathbb{R}_{+} \rightarrow \mathcal{C}$ is said to be resolution divergent if:

$$
\liminf _{\hat{\beta} \rightarrow \infty} \mathcal{P}\left(\left[\Sigma_{\mathcal{Y}_{0}}(\Delta(\beta)), \Gamma(\hat{\beta})\left(\mathcal{Y}_{\text {ref }}\right)\right]\right)=\infty, \quad \forall \beta \geq 0 .
$$

\footnotetext{
${ }^{1}$ We also admit the possibility that either $\mathcal{J}$ or $\mathcal{P}$ may not be defined for all their respective domains.
}

1) Basic Design: We define a controller as follows:

$$
\begin{aligned}
\Xi(\alpha, \phi)\left(y_{\text {ref }}\right): u & =-\hat{\theta}^{T} \phi(y)-x+y_{\text {ref }} \\
\dot{\hat{\theta}} & =\alpha D\left(\Omega_{0}, x\right) x \phi(y) \\
\hat{\theta}(0) & =0 \in \mathbb{R}^{p}
\end{aligned}
$$

Given a $\left(C(\Omega),\left\{K_{\beta}\right\}_{\beta \geq 0}\right)$ approximate model class $\left\{\phi^{m}\right\}_{m \geq 1}$, the control design is defined by:

$$
\begin{aligned}
\Gamma_{\text {Basic }}(\beta)(\cdot) & =\Xi\left(\alpha^{m}, \phi^{m}\right)(\cdot) \quad \text { where } \\
m & =\rho(\beta, \eta / 2), \alpha^{m}=\pi(m),
\end{aligned}
$$

for some function $\pi: \mathbb{N} \rightarrow \mathbb{R}_{+}$(the nature of $\pi$ will be considered in Section II-D.2). The stability result for this controller is given below, the proof is standard, but is given for completeness.

Theorem 2.6: There exists $\pi$ such that for all $\hat{\beta} \geq \beta$, the closed loop $\left[\Sigma_{\mathcal{Y}_{0}}(\Delta(\beta)), \Gamma_{\text {Basic }}^{\pi}(\hat{\beta})\left(\mathcal{Y}_{\text {ref }}\right)\right]$ is bounded and achieves asymptotic $\Omega_{0}$ tracking. Furthermore $\Gamma_{\text {Basic }}^{\pi}$ is $\mathcal{P}_{1}$, $\mathcal{P}_{2}, \mathcal{P}_{3}$ and $\mathcal{P}_{4}$ stable.

Proof: Let $f \in \Delta(\beta), y_{\text {ref }} \in \mathcal{Y}_{\text {ref }}, y_{0} \in \mathcal{Y}_{0}$. Let $\theta \in \mathcal{W}^{m}$ be such that: $\left\|f-\theta^{T} \phi^{m}\right\|_{C(\Omega)} \leq \frac{\eta}{2}$, whereby the existence of such a $\theta$ is guaranteed by definition of the dimension function $\rho$. Let $\pi: \mathbb{N} \rightarrow \mathbb{R}_{+}$be given by

$$
\pi(m)=\frac{\alpha^{*}}{\underline{\lambda}\left(R_{m}\right)}, \quad m \in \mathbb{N}
$$

where $R_{m}$ is the Gram matrix:

$$
\left(R_{m}\right)_{i j}=\left\langle\phi_{i}^{m}, \phi_{j}^{m}\right\rangle_{L^{2}(\Omega)},
$$

and where we will determine $\alpha^{*}>0$ subsequently.

Now consider the Lyapunov function

$$
V(x, \hat{\theta})=\frac{1}{2} x^{2}+\frac{1}{2 \pi(m)}(\theta-\hat{\theta})^{T}(\theta-\hat{\theta})
$$

and note that for $x \in \Omega \backslash \Omega_{0}$, it's derivative is given by:

$$
\dot{V}(x, \hat{\theta}) \leq-x^{2}+x\left(f-\theta^{T} \phi\right) \leq-x^{2}+\frac{|x| \eta}{2} .
$$

If $x(0) \in \Omega_{0}$ we claim that the $V(0)$ level set of $V$ is invariant, otherwise we claim that the $V(0)-\frac{1}{2} x_{0}^{2}+\frac{1}{2} \eta^{2}$ level set of $V$ is invariant. The proof of both claims is as follows. Let

$$
\delta_{2}=\left\|\frac{\delta_{\infty}}{w_{\infty}}\right\|_{L^{2}(\Omega)},
$$

so $\|f\|_{L^{2}(\Omega)} \leq \delta_{2}$. Let $t^{*}=\inf \left\{t \geq 0 \mid x(t) \in \partial \Omega_{0}\right\}$ if $x_{0} \in \Omega_{0}$, and $t^{*}=0$ otherwise. Then $V\left(t^{*}\right)$ is bounded as follows

$$
\begin{aligned}
V\left(t^{*}\right) & \leq \frac{1}{2} \max \left\{x_{0}^{2}, \eta^{2}\right\}+\frac{\theta^{T} \theta}{2 \pi(m)} \\
& \leq \frac{1}{2} \max \left\{x_{0}^{2}, \eta^{2}\right\}+\frac{\theta^{T} R \theta}{2 \pi(m) \underline{\lambda}\left(R_{m}\right)} \\
& =\frac{1}{2} \max \left\{\gamma_{0}^{2}, \eta^{2}\right\}+\frac{\left(\delta_{2}+\frac{W \eta}{2}\right)^{2}}{2 \alpha^{*}}=V_{0}
\end{aligned}
$$

from which it follows by inequality (11) that there exists $\alpha^{*}>0$ such that $\sqrt{2 V_{0}}+\gamma_{1}<W$ and hence

$$
\left[-\sqrt{2 V_{0}}-\gamma_{1}, \sqrt{2 V_{0}}+\gamma_{1}\right] \subset \Omega^{\circ} \text {. }
$$


It follows that $y(t) \in \Omega$ for all $t \geq 0$, and hence inequality (24) holds for all $t \geq 0$, provided we can show $\sqrt{2 V(t)}+\gamma_{1} \leq W$ for all $t \geq 0$, since $\frac{1}{2} x^{2}(t) \leq V(t)$ for all $t \geq 0$, and $|y(t)| \leq|x(t)|+\gamma_{1}$. For a contradiction suppose there exists $t^{* *} \geq 0$ such that $\sqrt{2 V\left(t^{* *}\right)}+\gamma_{1}=W$. It follows that:

$0 \leq \int_{T_{\eta} \cap\left[0, t^{*}\right]}-\dot{V} d t=V\left(t^{*}\right)-V\left(t^{* *}\right) \leq V_{0}-\frac{\left(W-\gamma_{1}\right)^{2}}{2}$

which contradicts the inequality $\sqrt{2 V_{0}}+\gamma_{1}<W$. This also shows that $V(t) \leq V_{0}$ for all $t \geq t^{*}$, and $V(t) \leq V_{0}$ for all $t \geq 0$ since for $t \in\left[0, t^{*}\right), \dot{\hat{\theta}}=0$ and $x^{2}(t) \leq \eta^{2}$.

Since $V(t) \leq V_{0}$ for all $t \geq 0$, it follows that $x(\cdot), \hat{\theta}(\cdot) \in L^{\infty}$ and by integrating inequality (24) we obtain:

$$
\int_{T_{\eta}} x^{2}(t) d t \leq \int_{T_{\eta}}-\dot{V} d t \leq V_{0}-V(\infty) \leq V_{0}{ }^{3}
$$

Since $x, \hat{\theta}$ is bounded, it follows from the system equation that $\dot{x}$ is bounded, hence $x$ is uniformly continuous. Then equation (28) impies $x(t) \rightarrow \Omega_{0}$. As $f \in \Delta(\beta), y_{\text {ref }} \in \mathcal{Y}_{\text {ref }}, y_{0} \in \mathcal{Y}_{0}$ were arbitrary, this completes the proof of boundedness and asymptotic $\Omega_{0}$ tracking. $\mathcal{P}_{1}, \mathcal{P}_{2}, \mathcal{P}_{3}$ and $\mathcal{P}_{4}$ stability is now established. First we obtain a bound on $m\left(T_{\eta}\right)^{4}$ as follows:

$$
\begin{aligned}
m\left(T_{\eta}\right) & =\int_{T_{\eta}} 1 d t \leq \int_{T_{\eta}} \frac{-\dot{V}}{\inf _{t \in T_{\eta}}|\dot{V}|} d t \\
& \leq \frac{2}{\eta^{2}} \int_{T_{\eta}}-\dot{V} d t \leq \frac{2 V(0)}{\eta^{2}} \leq \frac{2 V_{0}}{\eta^{2}} .
\end{aligned}
$$

Then we observe that the $L^{\infty}$ bounds on $x, \hat{\theta}$ can be written directly in terms of $V_{0}$. Since all terms in the cost functional $x, u, \dot{u}$ are functions of $x, \hat{\theta}, y_{\text {ref }}, \dot{y_{\text {ref }}}$, it follows that the cost can be bounded in terms of $V_{0}, \gamma_{1}, \gamma_{2}$. Since $V_{0}$ is in turn is bounded by construction in terms of $\gamma_{1}$ and $W$, this completes the proof.

2) Scaling of the adaption gain: The construction used in the proof above uses an adaption gain which scales $\pi(m)=$ $\frac{\alpha^{*}}{\underline{\lambda}\left(R_{m}\right)}$. For many approximants this is a divergent function (eg. for B-splines or for the Guassian RBF's of [2], see eg. [1]). However, although this is only a sufficient condition for stability, note that there are two reasons for scaling the adaption gain $\alpha^{m}$ : the first is to ensure stability, the second is to ensure resolution scaleability.

For many approximators, imposing the (sufficient) condition (26) forces $\alpha^{m}$ to be an increasing function of the resolution. For example, any approximate model class satisfying [1]:

$$
\exists \Upsilon_{2}>0, \text { s.t. } \forall m \geq 1, \sup _{y \in \Omega}\left|\phi^{m}(y)\right| \leq \Upsilon_{2},{ }^{5}
$$

has the property that for some $\epsilon>0,(\epsilon 1)^{T} \phi^{m} \in \Delta(\beta)$. Hence,

$$
V(0) \geq \frac{\epsilon^{2} 1^{T} 1}{2 \pi(m)} \geq \frac{\epsilon m}{2 \pi(m)}
$$

\footnotetext{
${ }^{3}$ Here we use the fact that $\hat{\theta}(t)$ is constant when $x(t) \in \Omega_{0}-$ see eg. [1] for details.

${ }^{4}$ Here, $m(A)$ denotes the Lebesgue measure of the set $A$.

${ }^{5}$ For examples, B-splines, and Guassian RBF's with the scaling of [2] have this property.
}

which implies $\pi$ is required to be s.t. $\pi(m) \geq O(m)$. In fact, for some classes of approximator, we can show that it is also neccessary for $\pi$ to be divergent for $x$ to remain in any bounded region. An example of this was given in [11].

Let us now consider the behaviour of $\int_{T_{\eta}} x^{2}(t) d t$ as $m$ increases, assuming $x$ is bounded by $\Omega$.

1) Again consider approximators with the property (30). In the limiting case where $\Omega_{0}=\{0\}$, (or even $\Omega_{0}=\emptyset$ ), sufficiently rich reference signals force a persistantly exciting regressor, and hence parameter convergence. Now consider the Lyapunov function (52) and integrate it's derivative, to obtain

$$
\int_{0}^{\infty} x^{2}(t) d t=V(0)-V(\infty)=\frac{1}{2} x_{0}^{2}+\frac{1}{2 \pi(m)} \theta^{T} \theta
$$

since $x(\infty)=0, \hat{\theta}(\infty)=\theta$. By again considering $\theta=\epsilon 1$, this shows that uniform boundedness of $\int_{T_{\eta}} x^{2}(t) d t$ occurs if and only if $\pi(m) \geq O(m)$.

2) For more limited classes of approximants, easy direct proofs of the neccessity of divergent scaling of the adaption gain can be given in the prescence of a deadzone. Here we illustrate the neccesity of such a scaling for a simple class of popular approximants, namely compactly supported mesh based approximants (eg. Bsplines on uniform lattices).

We consider approximants defined by: $\phi^{m}: \mathbb{R} \rightarrow \mathbb{R}^{2 m+1}$, where

$$
\phi^{m}=\left(\phi_{-m}^{m}, \phi_{-m+1}^{m}, \ldots, \phi_{m-1}^{m}, \phi_{m}^{m}\right)
$$

for $m \geq 1$ and where

$$
\phi_{i}^{m}(x)=F(m x-W i),
$$

where $F: \mathbb{R} \rightarrow \mathbb{R}$ is continuous, positive, compactly supported and has a maximum $F(0)>0$ at 0 .

It is straightforward to show that there exists constants $l, k \geq 1$ such that $\forall m \geq 1, \exists m / k$ intervals $I_{i}^{m} \subset[\eta, \min (W / 2, l F(0) / 2)]$ with the property that between 1 and $l$ basis functions $\phi_{i}^{m},-m \leq i \leq m$, intersect $I_{i}^{m}$ and furthermore

$$
\begin{aligned}
\operatorname{supp} \phi_{j} \cap I_{i}^{m} / & =\emptyset, \\
\operatorname{supp} \phi_{k} \cap I_{p}^{m} / & =\emptyset, \\
\operatorname{supp} \phi_{j} \cap \operatorname{supp} \phi_{k} / & =\emptyset \Rightarrow i=p .
\end{aligned}
$$

Now define $K_{i}^{m}=\left\{j \in[-m, m] \mid \operatorname{supp} \phi_{j}^{m} \cap I_{i} \neq \emptyset\right\}$, and let $J_{i}^{m}=\cup_{j \in K_{i}^{m}} \operatorname{supp} \phi_{j}^{m}$, note that $I_{i}^{m} \subset J_{i}^{m}$, and $J_{i}^{m} \cap J_{j}^{m}=\emptyset$ if $i \neq j$.

Let $\epsilon>0$ be such that

$$
\begin{aligned}
\epsilon^{2} F(0)^{2} W & \leq \delta_{2}^{2}, \quad \text { and } \\
\epsilon F(0) \sup _{y \in \Omega}\left|w_{\infty}(y)\right| & \leq \delta_{\infty},
\end{aligned}
$$

so if $\theta=\epsilon 1$ then $\theta^{T} \phi^{m} \in \Delta(\beta)$ for all $m \geq 1$. Consider $y_{\text {ref }}=0, y_{0}=\min (W / 2, \epsilon F(0) / 2)$, and $\bar{f}=(\epsilon 1)^{T} \phi^{m}$. Let $y_{i}^{* m}=\frac{W i}{m} \in I_{i}^{m}$, so $\phi_{i}^{m}\left(y_{i}^{* m}\right)=F(0)$. To drive $y(\cdot) \rightarrow \eta$ it is necessary to cross the point $y_{i}^{* m}$. For this 
to happen, $\dot{y} \leq 0$, ie. $(\theta-\hat{\theta})^{T} \phi^{m}\left(y^{*}{ }_{i}^{m}\right)-y_{i}^{* m} \leq 0$. Now

$$
0 \leq y_{i}^{* m} \leq \min (W / 2, \epsilon F(0) / 2),
$$

and

$$
\theta^{T} \phi^{m}\left(y_{i}^{* m}\right) \geq F(0) \epsilon,
$$

which implies that it is necessary for

$$
\hat{\theta}^{T} \phi^{m}\left(y_{i}^{* m}\right) \geq F(0) \epsilon / 2
$$

to cross $y_{i}^{* m}$. This implies that $\sum_{j \in K_{i}^{m}} \hat{\theta}_{j} \geq \epsilon / 2$. Since $\left|\dot{\hat{\theta}}_{i}\right| \leq \alpha W F(0)$ and $\dot{\hat{\theta}}_{i}=0$ if $y(t) \notin J_{i}^{m}$ it follows that the total time whilst $y(t) \in J_{i}$ is greater than $\frac{\epsilon}{2 \alpha W F(0) l}$. By the disjointness property of the $J_{i}$ 's and the fact that the $\dot{\hat{\theta}}_{i}=0$ if $y(t) \notin J_{i}^{m}$, it follows that

$$
m\left(T_{\eta}\right) \geq \frac{m \epsilon}{2 \alpha W F(0) l k},
$$

and hence

$\int_{T_{\eta}} x^{2}(t) d t=\int_{T_{\eta}} y^{2}(t) d t \geq \frac{m \epsilon \eta^{2}}{2 \alpha W F(0) l k} \geq O\left(\frac{m}{\alpha}\right)$.

This again shows that uniform boundedness of $\int_{T_{\eta}} x^{2}(t) d t$ occurs if and only if $\pi(m) \geq O(m)$.

The following result then motivates the rest of the paper:

Theorem 2.7: Suppose $\pi$ is divergent. Then the closed loop $\left[\Sigma_{\mathcal{Y}_{0}}(\Delta(\beta)), \Gamma_{\text {Basic }}^{\pi}(\hat{\beta})\left(\mathcal{Y}_{\text {ref }}\right)\right]$ is $\mathcal{P}_{3}$ and $\mathcal{P}_{4}$ resolution divergent.

Proof: See Section II-D.4, Theorem 2.9.

A corollary of this result is the statement that for the classes of approximators considered in above, there is no choice of $\pi$ for which resolution divergence can be avoided. The aim of the paper is therefore to examine the relative performances of two designs which have been shown to have resolution scaleability properties.

It remains an open question whether it is $\mathcal{P}_{1}$ or $\mathcal{P}_{2}$ resolution scaleable for any choice of approximator structure for this scalar system, although it seems likely for some wide classes of approximator that it is resolution divergent - see [1] for related results showing a similar performance divergence for MIMO equivalents of this controller and a discussion of this question.

3) Projection Modification: The projection modification is typically used to ensure boundedness of the parameter estimator when disturbances are present. It does not guarantee any form of convergence of the output. However, it has been proposed as a technique to ensure $\mathcal{P}_{1}$ resolution scaleability when used in conjunction with a dead-zone ${ }^{6}[8]$, which we now describe.

Suppose $\mathcal{W}$ is a convex set in $\mathbb{R}^{p}$ with a smooth boundary, written in the form:

$$
\mathcal{W}=\left\{\theta \in \mathbb{R}^{p}: \Pi(\theta) \leq 0\right\}
$$

\footnotetext{
${ }^{6}$ It is important to note that convergence of the output can be maintained when a dead-zone is used in conjunction with projection.
}

The controller $\Xi_{\text {Proj }}$ is the same as the basic controller (20), except for a change to the adaptive law:

$$
\begin{aligned}
\Xi(\alpha, \phi, \mathcal{W})\left(y_{\mathrm{ref}}\right): u & =-\hat{\theta}^{T} \phi(y)-x+y_{\mathrm{ref}} \\
\dot{\hat{\theta}} & =\alpha D\left(\Omega_{0}, x\right) \operatorname{Proj}_{\mathcal{W}}(x \phi(y)) \\
\hat{\theta}(0) & =0 \in \mathbb{R}^{p} .
\end{aligned}
$$

In this definition the projection operator $\operatorname{Proj}_{\mathcal{W}}: \mathbb{R}^{p} \rightarrow \mathbb{R}^{p}$ is defined as follows:

$\operatorname{Proj}_{\mathcal{W}}(\tau)= \begin{cases}\tau, & \text { if } \hat{\theta} \in \Pi^{\circ} \text { or } \nabla \Pi^{T} \tau \leq 0 \\ \left(I-\frac{\nabla \Pi \nabla \Pi^{T}}{\nabla \Pi^{T} \nabla \Pi}\right) \tau & \text { if } \hat{\theta} \in \partial \Pi, \nabla \Pi^{T} \tau>0\end{cases}$

Given a $\left(C(\Omega),\left\{K_{\beta}\right\}_{\beta \geq 0}\right)$ approximate model class $\left\{\phi^{m}\right\}_{m \geq 1}$, the projection modified control design is taken to be:

$$
\Gamma_{\text {Proj }}(\beta)(\cdot)=\Xi\left(\alpha^{m}, \phi^{m}, \mathcal{W}^{m}\right)(\cdot)
$$

where $p=\operatorname{dim} \phi^{m}$, and

$$
\begin{aligned}
\mathcal{W}^{m} & =\left\{\theta \in \mathbb{R}^{p}|| \theta^{T} \phi^{m}(y) \mid \leq \delta_{\infty} w_{\infty}(y), \forall y \in \mathbb{R}\right\},{ }^{7} \\
m & =\rho(\beta, \eta / 2), \\
\alpha^{m} & =\pi(m) .
\end{aligned}
$$

We also impose a uniform strength condition [1]:

$$
\exists \Upsilon_{1}>0 \quad \text { s.t. } \Upsilon_{1} \leq \inf _{y \in \Omega}\left|\phi^{m}(y)\right| .
$$

This condition is satisfied by most approximants, see eg. [1].

The motivation for the introduction of the projection modification is the following result which shows that the design also additionally guarantees resolution scaleability w.r.t. both $\mathcal{P}_{1}$ and $\mathcal{P}_{2}$.

Theorem 2.8: There exists $\pi$ such that for all $\hat{\beta} \geq \beta$, the closed loop $\left[\Sigma_{\mathcal{Y}_{0}}(\Delta(\beta)), \Gamma_{\text {Proj }}^{\pi}(\hat{\beta})\left(\mathcal{Y}_{\text {ref }}\right)\right]$ is bounded and achieves asymptotic $\Omega_{0}$ tracking. $\Gamma_{\text {Proj }}^{\pi}$ is both $\mathcal{P}_{1}, \mathcal{P}_{2}, \mathcal{P}_{3}$ and $\mathcal{P}_{4}$ stable and $\mathcal{P}_{1}, \mathcal{P}_{2}$ resolution scaleable.

Proof: Let $f \in \Delta(\beta), y_{\text {ref }} \in \mathcal{Y}_{\text {ref }}, y_{0} \in \mathcal{Y}_{0}$. As in Theorem 2.6, take $\theta \in \mathcal{W}^{m}$ to be such that

$$
\left\|f-\theta^{T} \phi^{m}\right\|_{C(\Omega)} \leq \frac{\eta}{2},
$$

and observe that $\theta \in \mathcal{W}^{m}$ by construction. The proof of boundedness, asymptotic $\Omega_{0}$ tracking and $\mathcal{P}_{1}, \mathcal{P}_{2}$ stability, follows that of Theorem 2.6, by noting that inequality (24) can be established by the property [12]:

$$
(\theta-\hat{\theta})^{T} \operatorname{Proj}(\tau) \geq(\theta-\hat{\theta})^{T} \tau, \quad \forall \theta \in \mathcal{W}^{m},
$$

which holds with $\tau=\alpha x \phi(x)$ since $\theta(0)=0 \in \mathcal{W}^{m}$. The rest of the proof is analagous. To establish $\mathcal{P}_{1}$ and $\mathcal{P}_{2}$ resolution scaling, it suffices to establish bounds on $\|y\|_{L^{\infty}},\|u\|_{L^{\infty}}$ and $m\left(T_{\eta}\right)$ which are independant of $\hat{\beta}$ (and $m$ ). First we establish a bound on $V_{0}$ which is independant of $\hat{\beta}$. Taking the same choice for $\pi$ as in Theorem 2.6, $\pi(m)=\frac{\alpha^{*}}{\underline{\lambda}\left(R_{m}\right)}, \alpha^{*}>0$, we obtain the following uniform bound:

$$
\begin{aligned}
V_{0} & \leq \frac{1}{2} \max \left\{x_{0}^{2}, \eta^{2}\right\}+\frac{\left(\delta_{2}+\frac{\eta W}{2}\right)^{2}}{2 \pi(m) \underline{\lambda}\left(R_{m}\right)} \\
& =\frac{1}{2} \max \left\{\gamma_{0}^{2}, \eta^{2}\right\}+\frac{\left(\delta_{2}+\frac{\eta W}{2}\right)^{2}}{2 \alpha^{*}} .
\end{aligned}
$$


From the invariance of the $V_{0}$ level set of $V$, it follows that:

$$
\begin{aligned}
|y(t)| & \leq|x(t)|+\left|y_{\mathrm{ref}}(t)\right| \\
& \leq \sqrt{2 V(t)}+\gamma_{1} \leq \sqrt{2 V_{0}}+\gamma_{1} \\
|u(t)| & =\left|-\hat{\theta}^{T} \phi^{m}(y)-x-y_{\mathrm{ref}}\right| \\
& \leq\left|\delta_{\infty} w_{\infty}(y)\right|+|x|+\left|y_{\mathrm{ref}}\right| \\
& \leq\left|\delta_{\infty} w_{\infty}\left(\sqrt{2 V_{0}}+\gamma_{1}\right)\right|+\sqrt{2 V_{0}}+\gamma_{2} .
\end{aligned}
$$

A bound on $m\left(T_{\eta}\right)$ is established as in Theorem 2.6: $m\left(T_{\eta}\right) \leq \frac{2 V_{0}}{\eta^{2}}$. Since in all the above inequalities, $f \in \Delta(\beta)$, $y_{\text {ref }} \in \mathcal{Y}_{\text {ref }}, y_{0} \in \mathcal{Y}_{0}$ were arbitrary, the result follows.

4) The Resolution Divergence Properties of the Projection Modification: In this section we establish the poor scaling properties of the basic and projection modified designs when there is an additional penalty on the control rate.

Theorem 2.9: Suppose $\pi$ is divergent. Then $\Gamma_{\text {Basic }}^{\pi}, \Gamma_{\text {Proj }}^{\pi}$ are both $\mathcal{P}_{3}$ and $\mathcal{P}_{4}$ stable and $\mathcal{P}_{3}$ and $\mathcal{P}_{4}$ resolution divergent.

Proof: $\mathcal{P}_{3}$ and $\mathcal{P}_{4}$ stability has been established in Theorem 2.6 and Theorem 2.8. To establish resolution divergence, first consider $\Gamma_{\text {Proj }}^{\pi}$. Choose $y_{\text {ref }} \in \mathcal{Y}_{\text {ref }}, y_{0} \in \mathcal{Y}_{0}$ such that $y_{\mathrm{ref}}(0)=\ddot{y_{\mathrm{ref}}}(0)=\ddot{y_{\mathrm{ref}}}(0)=0, y_{0}=\frac{W-\eta}{2}$, so we have $x(0) \notin \Omega_{0}$. Then:

$$
\dot{u}(0)=\alpha x_{0} \phi^{m}\left(y_{0}\right)^{T} \phi^{m}\left(y_{0}\right)-x_{0}+f\left(y_{0}\right) .
$$

since $\hat{\theta}(0)=0$. Since $\phi^{m}\left(y_{0}\right)^{T} \phi^{m}\left(y_{0}\right) \geq \Upsilon_{1}$ it follows that $|\dot{u}(0)| \rightarrow \infty$ as $\alpha \rightarrow \infty$. In particular, if $\pi$ is divergent, then $\Gamma_{\text {Proj }}^{m}$ is $\mathcal{P}_{3}, \mathcal{P}_{4}$ resolution divergent. The proof for $\Gamma_{\text {Basic }}$ is similar.

5) Multi-resolution Design: Define a controller as follows:

$$
\begin{aligned}
\Xi(\alpha, G, r, \phi)\left(y_{\text {ref }}\right): u & =-\hat{\theta}^{T} \phi(y)-x+y_{\text {ref }} \\
\dot{\hat{\theta}} & =\alpha D\left(\Omega_{0}, x\right) x G \phi(y), \\
\hat{\theta}(0) & =0 \in \mathbb{R}^{p}
\end{aligned}
$$

The adaptive structure matric $G$ is taken to be positive definite and symmetric. The multi-resolution (definition 2.1) model class $\left\{\phi^{m}\right\}_{m \geq 1}$ is assumed to be a $\left\{K_{\beta}\right\}_{\beta \geq 0}$ approximate model class and the control design is defined by:

$\Gamma(\beta)(\cdot)=\Xi\left(\alpha^{*}, G^{m}, \phi^{m}\right)(\cdot), \quad$ where $\quad m=\rho(\beta, \eta / 2)$,

and where $\left\{G^{m}, \phi^{m}\right\}_{m \geq 1}$ are 1-regular pairs, in the sense of the following definition [5]:

Definition 2.10: A model class $\left\{\phi^{m}\right\}_{m \geq 1}$ and a sequence of adaptive structure matrices $\left\{G^{m}\right\}_{m \geq 1}$ is said to be a $k$ regular pair if there exists a continuous function $\mu: \mathbb{R} \rightarrow \mathbb{R}_{+}$ such that for all $m \geq 1$

$$
\left\|D^{\zeta}\left(G^{m}\right)^{\frac{1}{2}} \phi^{m}(x)\right\| \leq \mu(x), \quad \forall x \in \mathbb{R}, \quad 0 \leq \zeta \leq k .
$$

Here $D^{\zeta}$ denotes the differential operator $D^{\zeta}=\frac{\partial^{\zeta}}{\partial x^{\zeta}}$. Construction of suitable matrices $G^{m}$ is straightforward and can be found in [1], [5]. We can then give the following result:

Theorem 2.11: There exists $\alpha^{*}>0$ such that for all $\hat{\beta} \geq \beta$, the closed loop $\left[\Sigma_{\mathcal{Y}_{0}}(\Delta(\beta)), \Gamma_{\mathrm{mr}}(\hat{\beta})\left(\mathcal{Y}_{\text {ref }}\right)\right]$ is bounded and achieves asymptotic $\Omega_{0}$ tracking. $\Gamma_{\mathrm{mr}}$ is both $\mathcal{P}_{1}, \mathcal{P}_{2}, \mathcal{P}_{3}$ and $\mathcal{P}_{4}$ stable and $\mathcal{P}_{1}, \mathcal{P}_{2}, \mathcal{P}_{3}$ and $\mathcal{P}_{4}$ resolution scaleable.

Proof: Boundedness, $\Omega_{0}$ asymptotic tracking, $\mathcal{P}_{1}, \mathcal{P}_{2}$ stability and $\mathcal{P}_{1}$ resolution scaleability has been established previously [11]. A complete proof of $\mathcal{P}_{1}, \mathcal{P}_{2}, \mathcal{P}_{3}, \mathcal{P}_{4}$ resolution scaleability is as follows.

Since $\Delta(\beta) \subset K_{\beta}$, there exists $M \geq 1$, such that for all $f \in \Delta(\beta)$, there exists $\theta^{M} \in \mathcal{W}^{M}$ such that

$$
\left\|f-\left(\theta^{M}\right)^{T} \phi^{M}\right\|_{C(\Omega)} \leq \frac{\eta}{2}
$$

hence it follows that $\theta=\left[\left(\theta^{M}\right)^{T} \mid 0\right]^{T}$ has the property that:

$$
\left\|f-(\theta)^{T} \phi^{m}\right\|_{C(\Omega)} \leq \frac{\eta}{2}
$$

since $\phi^{m}$ is a multi-resolution model. Now let $y_{\text {ref }} \in \mathcal{Y}_{\text {ref }}$, $y_{0} \in \mathcal{Y}_{0}$ and consider the previous Lyapunov function (52)

$$
V(x, \hat{\theta})=\frac{1}{2} x^{2}+\frac{1}{2 \alpha^{*}}(\theta-\hat{\theta})^{T}(\theta-\hat{\theta})
$$

Since $\left|\theta^{M}\right|$ is bounded independently of $m$, it follows that $|\theta|$ is bounded independently of $m$ and hence analagously to Theorem $2.8 V_{0}$ is uniformly bounded independantly of $\hat{\beta}$. Analagously to Theorem 2.8, since the $V_{0}$ level set is invariant, it can also be shown that $m\left(T_{\eta}\right),\|x\|_{L^{\infty}}$ are uniformly bounded in terms of $V_{0}$. It thus suffices to show that $u, \dot{u}$ are uniformly bounded independant of $\hat{\beta}$. First we establish two inequalities:

$$
|u(t)|=\left|-\hat{\theta}^{T} \phi(y)-x+\dot{y_{\text {ref }}}\right| \leq\left|\hat{\theta}^{T} \phi(y)\right|+|x|+\left|\dot{y_{\text {ref }}}\right|,
$$

$$
\begin{aligned}
|\dot{u}(t)|= & \left|-(\dot{\hat{\theta}})^{T} \phi(y)-\hat{\theta}^{T} \frac{\partial \phi}{\partial y} \dot{y}-\dot{x}+\ddot{y_{\mathrm{ref}}}\right| \\
= & \mid-\alpha x \phi(y)^{T} G \phi(y)-\hat{\theta}^{T} \frac{\partial \phi}{\partial y}\left(\dot{x}+\dot{y_{\mathrm{ref}}}\right) \\
& +x-f(y)+\hat{\theta}^{T} \phi(y)+\ddot{y_{\mathrm{ref}}} \mid \\
\leq & \left|\alpha x \phi(y)^{T} G \phi(y)\right| \\
& +\left|\hat{\theta}^{T} \frac{\partial \phi}{\partial y}\right|\left(|x|+|f(y)|+\left|\hat{\theta}^{T} \phi(y)\right|+\left|y_{\mathrm{ref}}\right|\right) \\
& +|x|+|f(y)|+\left|\hat{\theta}^{T} \phi(y)\right|+\left|\ddot{y_{\mathrm{ref}}}\right| .
\end{aligned}
$$

Since $\|x\|_{L}^{\infty},\|y\|_{L^{\infty}},\|f(y)\|_{L^{\infty}}, \quad y_{\text {ref }}, \quad \dot{y_{\text {ref }}}, \ddot{y_{\text {ref }}}$ can be bounded uniformly by $V_{0}, \gamma_{0}, \gamma_{1}, \gamma_{2}, \delta_{\infty}$, it suffices to bound $\left\|\phi(y)^{T} G \phi(y)\right\|_{L^{\infty}}\|\hat{\theta} \phi(y)\|_{L^{\infty}},\left\|\hat{\theta} \frac{\partial \phi(y)}{\partial y}\right\|_{L^{\infty}}$ independantly of $\hat{\beta}$. Clearly, $\left|\phi(y)^{T} G \phi(y)\right| \leq \mu^{2}(y)$ and by letting $D$ denote either $D^{0}$ or $D^{1}$, we have:

$$
\begin{aligned}
\left|\hat{\theta}^{T} D \phi^{m}\right| \leq & \left|(\theta-\hat{\theta})^{T} D \phi^{m}\right|+\left|\theta^{T} D \phi^{m}\right| \\
\leq & \sqrt{(\theta-\hat{\theta})^{T}\left(G^{m}\right)^{-1}(\theta-\hat{\theta})}\left\|D\left(G^{m}\right)^{\frac{1}{2}} \phi^{m}\right\| \\
& +\sqrt{\theta^{T}\left(G^{m}\right)^{-1}} \theta \mid D\left(G^{m}\right)^{\frac{1}{2}} \phi^{m} \| \\
\leq & \sqrt{2 \alpha^{*} V(x, \hat{\theta})} \mu(y)+\sqrt{2 \alpha^{*} V(x(0), 0)} \mu(y) \\
\leq & 2 \mu(y) \sqrt{2 \alpha^{*} V_{0}} .
\end{aligned}
$$

Since $y$ is uniformly bounded as a function of $V_{0}$, this completes the proof. 


\section{E. Comparison between Designs}

The main result in this paper shows that when the control rate is penalised in the cost functional and when the apriori estimate $\hat{\beta}$ of the smoothness $\beta$ becomes conservative, then the multi-resolution design increasingly out-performs the projection modified design.

Theorem 2.12: Suppose $\pi$ is divergent, then for $i=3,4$ :

$$
\frac{\mathcal{P}_{i}\left[\Sigma_{\mathcal{Y}_{0}}(\Delta(\beta)), \Gamma_{\text {Proj }}^{\pi}(\hat{\beta})\left(\mathcal{Y}_{\text {ref }}\right)\right]}{\mathcal{P}_{i}\left[\Sigma_{\mathcal{Y}_{0}}(\Delta(\beta)), \Gamma_{\mathrm{mr}}(\hat{\beta})\left(\mathcal{Y}_{\text {ref }}\right)\right]} \rightarrow \infty \quad \text { as } \quad \hat{\beta} \rightarrow \infty
$$

Proof: This is a simple consequence of the resolution divergence property of $\Gamma_{\text {Proj }}^{\pi}$ (Theorem 2.9) and the resolution scaling property of $\Gamma_{\mathrm{mr}}^{\pi}$ (Theorem 2.11).

Corollary 12.1: For the class of systems given in Section II-D.2, number II-D. 2 we have for $i=3,4$ :

$$
\frac{\inf _{\pi} \mathcal{P}_{i}\left[\Sigma_{\mathcal{Y}_{0}}(\Delta(\beta)), \Gamma_{\text {Proj }}^{\pi}(\hat{\beta})\left(\mathcal{Y}_{\text {ref }}\right)\right]}{\mathcal{P}_{i}\left[\Sigma_{\mathcal{Y}_{0}}(\Delta(\beta)), \Gamma_{\mathrm{mr}}(\hat{\beta})\left(\mathcal{Y}_{\text {ref }}\right)\right]} \rightarrow \infty \quad \text { as } \quad \hat{\beta} \rightarrow \infty
$$

Proof: For the class of systems considered, we have shown that it is neccessary for $\pi$ to be divergent to achieve resolution scaleability of the $x^{2}$ term in the cost. Hence by Theorem 2.12, the corollary follows.

\section{F. Extensions}

For notational simplicity we have only considered the simplest case of a scalar system with a matched nonlinearity. However, these results can straightforwardly be extended to the case of the integrator chain:

$$
y^{(n)}=f\left(y, \dot{y}, \ldots, y^{(n-1)}\right)+u
$$

by the obvious alterations to the basic control law:

$$
\begin{aligned}
& \Xi(\alpha, r, \phi)\left(y_{\mathrm{ref}}\right): \\
u= & -\hat{\theta}^{T} \phi\left(y, \dot{y}, \ldots, y^{(n-1)}\right)-a^{T} x+\dot{y}_{\mathrm{ref}}^{(n)} \\
\dot{\hat{\theta}}= & \alpha D\left(\Omega_{0}, x\right) x^{T} b \phi\left(y, \dot{y}, \ldots, y^{(n-1)}\right) \\
\hat{\theta}(0)= & 0 \in \mathbb{R}^{p} \\
x & =\left(y-y_{\mathrm{ref}}, \dot{y}-y_{\mathrm{ref}}, \ldots, y^{(n-1)}-y_{\mathrm{ref}}^{(n-1)}\right)(58
\end{aligned}
$$

where

$$
\Omega_{0}=\left\{x \in \mathbb{R}^{n} \mid x^{T} P x \leq \eta\right\}
$$

and with appropriate choices for $a, b$ and $P=P^{T}>0$, see [5] for details. Similar arguments establish bounded asymptotic $\Omega_{0}$ tracking and resolution divergence of $\Gamma_{\text {Basic }}, \Gamma_{\text {Proj }}$ and the resolution scaleability of $\Gamma_{\mathrm{mr}}$, by considering the Lyapunov function:

$$
V(x, \hat{\theta})=x^{T} P x+\frac{1}{2 \alpha}(\theta-\hat{\theta})^{T} G^{-1}(\theta-\hat{\theta}) .
$$

The results can also be extended to strict feedback systems, note that the results in [5] construct resolution scaleable controllers of a multi-resolution type for $\mathcal{P}_{1}$, (and can be easily extended to $\mathcal{P}_{2}, \mathcal{P}_{3}, \mathcal{P}_{4}$ ). Resolution divergence of projection modified designs [13] remain to be established, but it can be expected that resolution divergence can even be obtained w.r.t.
$\mathcal{P}_{2}$ since the control $u$ is directly dependant on the adaption gain $\alpha$.

Resolution scaleability of the multi-resolution design can be established with respect to cost functionals incorporating penalties on higher derivatives, eg. terms such as:

$$
\int_{T_{\eta}}\left(u^{(k)}(t)\right)^{2} d t \quad \text { or } \quad \sup _{t \geq 0}\left|u^{(k)}(t)\right|,
$$

by demanding on the k-regularity of the pair $\left(G^{k}, \phi^{k}\right)_{k \geq 0}$, and by further constraining the reference trajectory to lie in $Y_{k}^{\text {ref }}$, which is defined by:

$$
\begin{gathered}
Y_{k}^{\text {ref }}=\left\{y_{\text {ref }} \in C\left(\mathbb{R}_{+}, \mathbb{R}\right) \mid\right. \\
\left.\left\|y_{\text {ref }}{ }^{i}\right\|_{L^{\infty}} \leq \gamma_{i+1}<\infty, 0 \leq i \leq k\right\} .(61) \\
\text { III. ConCLUSIONS }
\end{gathered}
$$

\section{CONCLUSIONS}

We have compared the two main proposals for resolution scaling, and showed the multi-resolution approximant idea is superior when higher derivatives of the control are penalised. For reasons of notational simplicity we have considered scalar systems, but have outlined how the arguments apply to much wider classes of systems. The multi-resolution results can be interpreted as showing how to select appropriate function approximators and adaption rates to avoid high gains; such high gains do occur as the resolution of some standard approximators are increased. 


\section{Notation and Nomenclature}

$\mathcal{W}^{m}$

$\phi^{m}: \mathbb{R} \rightarrow \mathcal{W}^{m}$

$\left\{\phi^{m}: \mathbb{R} \rightarrow \mathcal{W}^{m}\right\}_{m \geq 1}$

$\left\{\tilde{\phi}^{k}\right\}_{k \geq 1}: \mathbb{R} \rightarrow \mathcal{W}^{m}$

$\epsilon$

$\theta$

$\hat{\theta}$

$\rho$

$R_{m}$

$\Upsilon_{1}, \Upsilon_{2}$

$f: \mathbb{R} \rightarrow \mathbb{R}$

$\beta$

$\hat{\beta}$

$\left\{K_{\beta}\right\}_{\beta \geq 0}$

$\Delta(\beta)$

$w_{\infty}, \delta_{\infty}$

$\Sigma$

$\Xi$

$[\Sigma, \Xi]$

$\mathcal{U}$

$\mathcal{Y}$

$\mathcal{Y}_{0}$

$\gamma_{0}$

$\mathcal{Y}_{\text {ref }}$

$\gamma_{1}, \gamma_{2}$

$y_{\text {ref }}$

$u, y, x$

$C(\Omega)$

$C^{1}(\Omega)$

$L^{\infty}\left(\Omega ; w_{\infty}\right)$

$\Omega_{0}$

$T_{\Omega_{0}}$

$\pi: \mathbb{N} \rightarrow \mathbb{R}_{+}$

$\Gamma, \Gamma_{\text {Basic }}^{\pi}, \Gamma_{\text {Proj }}^{\pi}, \Gamma_{\mathrm{mr}}$

$\mathcal{J}, \mathcal{J}_{1}, \mathcal{J}_{2}, \mathcal{J}_{3}, \mathcal{J}_{4}$

$\mathcal{P}, \mathcal{P}_{1}, \mathcal{P}_{2}, \mathcal{P}_{3}, \mathcal{P}_{4}$ approximator size,

parameter space,

model base,

model base class,

base class,

approximation error,

model parameter,

adaptive estimate of model parameter,

dimension function,

Gram matrix of model $\phi^{m}$,

upper and lower strength bounds,

plant nonlinearity,

function smoothness parameter,

a-priori bound on the function

smoothness parameter,

smoothness class,

uncertainty set,

$L^{\infty}$ weight and bound,

plant,

controller,

plant, controller interconnection,

space of input signals,

space of output signals,

set of initial conditions,

bound on initial condition size,

set of reference signals,

bounds on reference signal size,

reference signal,

input, output and tracking error signal, space of continuous functions $\Omega \rightarrow \mathbb{R}$, space of continuously differentiable functions $\Omega \rightarrow \mathbb{R}$, weighted $L^{\infty}$ space, the dead-zone region $[-\eta, \eta], \eta>0$, time set when $x$ is not in dead-zone, adaptive gain scaling function,

control design mappings,

cost functionals,

worst case cost functionals.

\section{REFERENCES}

[1] M. French, C. Szepesvári, and E. Rogers, "An asymptotic scaling analysis of LQ performance for an approximate adaptive control design," Mathematics of Control, Signals and Systems, vol. 15, no. 2, pp. 145176, 2002.

[2] R. Sanner and J.-J. Slotine, "Gaussian networks for direct adaptive control," IEEE Trans. on Neural Networks, vol. 3, no. 6, pp. 837-863, 1992.

[3] E. Tzirkel-Hancock, "Stable control of nonlinear systems using neural networks," Ph.D. dissertation, Department of Engineering, University of Cambridge, 1992.

[4] L. F.L., J. S., and A. Yesildirak, Neural Network Control of Robot Manipulators and Non-Linear Systems. CRC Press, 1999.

[5] M. French, C. Szepesvari, and E. Rogers, Performance of Nonlinear Approximate Adaptive Controllers. Wiley, 2003.

[6] S. Ge, C. Hang, T. Lee, and T. Zhang, Stable Adaptive Neural Network Control. Kluwer, 2002.

[7] S. Ge and C. Wang, "Adaptive neural control of uncertain mimo nonlinear systems," IEEE Trans. on Neural Networks, vol. 15, no. 3, pp. 674-692, 2004.

[8] M. French, "Adaptive control of functionally uncertain systems," Ph.D. dissertation, University of Southampton, UK, 1998.

[9] M. Polycarpou and P. Ioannou, "On the existence and uniqueness of solutions in adaptive control systems," IEEE Trans. on Automatic Control, vol. 38, no. 3, pp. 474-479, 1993.

[10] M. French, "An analytical comparison between the weighted LQ performance of a robust and an adaptive backstepping design," IEEE Trans. on Automatic Control, vol. 47, no. 4, 2002.

[11] M. French, C. Szepesvári, and E. Rogers, "Uncertainty, performance and model dependency in approximate adaptive nonlinear control," IEEE Trans. on Automatic Control, vol. 45, no. 2, pp. 353-58, 2000.

[12] M. Krstić, I. Kanellakopoulos, and P. Kokotović, Nonlinear and Adaptive Control Design, 1st ed. New York: Wiley, 1995.

[13] R. Freeman, M. Krstić, and P. Kokotović, "Robustness of adaptive nonlinear control to bounded uncertainties," Automatica, vol. 34, no. 10, pp. 1227-1230, 1998.

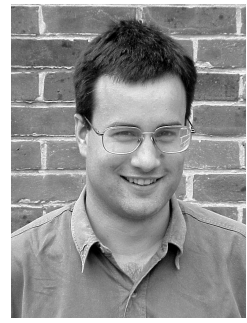

Mark French (S96, M98) read mathematics at Oxford University, and obtained his Ph.D. from the University of Southampton in 1998. He is currently a senior lecturer in the School of Electronics and Computer Science, University of Southampton. His current research interests are in the area of robust nonlinear control and robust adaptive control. He serves as an associate editor of the International Journal of Control. 